\title{
DOENÇA DE CUSHING E HIPERTENSÃO ARTERIAL: RELATO DE CASO
}

\section{CUSHING DISEASE AND HYPERTENSION: A CASE REPORT}

Vinicius Nasser', Daniel Catto De Marchi', Fernanda R Bezerra de Oliveira', Valéria Machado², Fernando Focaccia Póvoa², Maria Teresa Nogueira Bombig², Rui Póvoa²

\section{RESUMO}

Caso clínico de VNS, 23 anos, sexo feminino, branca, solteira, estudante, natural e procedente de São Paulo-SP com queixa de "pressão alta" há 4 anos. A paciente foi encaminhada para consulta ambulatorial após ter sido atendida em pronto-socorro (PS) com cefaleia, tonturas e pressão arterial (PA) 210x110 mm Hg e medicada com losartana 50 mg a cada 12 horas e hidroclorotiazida $25 \mathrm{mg}$ ao dia. Na avaliação ambulatorial relatava episódios prévios de cefaleia holocraniana de forte intensidade, latejante, de início gradual há 4 anos com piora há 2 anos, que a levava ao pronto-socorro (PS) com muita frequência. Além disso, referia aumento de peso e fraqueza generalizada. Ao exame físico apresentava-se com níveis elevados da PA 160x100 mm Hg em uso das medicações prescritas no PS, obesidade grau III (IMC 41), adiposidade localizada (giba), acantose nigricans e estrias violáceas abdominais. Foi diagnosticada a doença de Cushing associada à hipertensão arterial não controlada, realizados ajustes dos agentes anti-hipertensivos (associado anlodipino 5 mg a cada 12 horas) e solicitados exames laboratoriais. A monitorização ambulatorial da PA (MAPA) de 24 horas caracterizou a hipertensão resistente, as dosagens de cortisol (cortisol salivar $=8 \mathrm{~h}: 172$; após 23h:280, supressão com dexametasona $=<2,5$ ) e a ressonância nuclear magnética evidenciou um macroadenoma da hipófise confirmando o diagnóstico da doença de Cushing com base nos achados do exame físico, laboratorial e de imagem. A paciente foi tratada clinicamente com cetoconazol via oral sem sucesso e após isso submetida a ressecção transesfenoidal do macroadenoma de hipófise. Após o tratamento cirúrgico, houve perda de peso e resolução da hipertensão confirmada pelas medidas ambulatoriais de consultório e pela MAPA.

Descritores: Hipertensão; Hipófise; Monitorização da Pressão Arterial; Síndrome de Cushing.

\section{ABSTRACT}

Clinical case of VNS, 23 years old, female, white, single, student, born and living in São Paulo-SP, complaining of "high blood pressure" 4 years ago. The patient was referred to an outpatient clinic after being seen in the emergency room (ER) with headache, dizziness and blood pressure (BP) $210 \times 110 \mathrm{~mm} \mathrm{Hg}$ and medicated with losartan 50 mg every 12 hours and hydrochlorothiazide $25 \mathrm{mg}$ daily. In the outpatient evaluation, she reported previous episodes of severe, throbbing holocranial headache, which started gradually over 4 years and worsened over 2 years ago, which took her to the ER very often. In addition, she reported weight gain and generalized weakness. On physical examination, he presented with high BP levels $160 \times 100 \mathrm{~mm} \mathrm{Hg}$ using the medications prescribed in ER, obesity grade III (BMI 41), localized adiposity (gib), acanthosis nigricans and abdominal violet streaks. Cushing's syndrome was diagnosed associated with uncontrolled arterial hypertension, adjustments were made to antihypertensive agents (associated with amlodipine 5 mg every 12 hours) and laboratory tests were requested. 24-hour ambulatory BP monitoring (ABPM) characterized resistant hypertension, cortisol levels (salivary cortisol = 8:00 am: 172; after 11:00 pm: 280, dexamethasone suppression $=<2.5$ ) and magnetic resonance imaging revealed a macroadenoma of the pituitary gland confirming the diagnosis of Cushing's disease based on the findings of the physical, laboratory and imaging exam. The patient was clinically treated with oral ketoconazole and underwent transphenoid resection of the pituitary macroadenoma. After the surgery the patient was no longer hipertensive, without use of antihypertensive medications, outpatient office measurements were normal, as were measurements outside the office by ABPM.

Keywords: Hypertension; Pituitary; Ambulatory Blood Pressure Monitoring, Cushing's Syndrome.

1. Disciplina de Cardiologia da UNIFESP, São Pualo, SP, Brasil.

2. Cardiopatia Hipertensiva da Disciplina de Cardiologia da UNIFESP, São Pualo, SP, Brasil.

Correspondência: Vinicius Nasser de Carvalho. Setor de Cardiopatia Hipertensiva da Disciplina de Cardiologia da Universidade Federal de São Paulo (UNIFESP), Rua Loefgren 1350, Vila Clementino, São Paulo, SP, CEP O4O4O-001. vinicius.nasser@unifesp.br

http://dx.doi.org/10.47870/1519-7522/2021280148-53 


\section{INTRODUÇÃO}

A síndrome de Cushing (SC) é um distúrbio causado pelo excesso de cortisol associado à deficiência do mecanismo de regulação do eixo hipotálamo - hipófise - adrenal e do ritmo circadiano de secreção de cortisol. ${ }^{1}$ É caraterizada por um grupo de sinais e sintomas causados por uma exposição prolongada e inapropriada ao cortisol. ${ }^{2}$ A causa mais comum é o uso de glicocorticoides exógenos, porém pode surgir secundários a tumores adrenais com produção autônoma de cortisol (adenoma benigno ou maligno), hiperplasia adrenal, produção excessiva de hormônio adrenocorticotrópico (ACTH) ou tumor ectópico. ${ }^{2}$ Os principais sinais e sintomas observados na SC são obesidade central, fácies em lua cheia, estrias, hirsutismo, fraqueza muscular e diminuição da libido. A prevalência de hipertensão arterial (HA) foi encontrada em $80 \%$ dos adultos e $47 \%$ das crianças. ${ }^{2}$

A hipertensão na SC aparece precocemente, é um fator de risco isolado para mortalidade. ${ }^{3}$ e deve ser lembrada principalmente quando surge em jovens e em casos de hipertensão arterial resistente (HAR).

Diante de quadro clínico de hipercortisolismo deve-se fazer a diferenciação entre SC dependente de ACTH (80\%) e não dependente de $\mathrm{ACTH}(20 \%)^{3}$ sendo esta representada por doenças nas adrenais, principalmente adenomas, hiperplasias e carcinomas e que se caracterizam pela supressão do ACTH hipofisário em baixas concentrações (geralmente $<10$ $\mathrm{pg} / \mathrm{mL}){ }^{4,5}$ Dentre as SC dependentes de ACTH, o adenoma hipofisário gerando a doença de Cushing é o mais comum (90\%), porém os tumores ectópicos produtores de ACTH (10\%); tumor carcinoide brônquico, carcinoma de células pequenas, feocromocitoma e tumor de timo fazem parte do diagnóstico diferencial.

A doença de Cushing (DC), ou síndrome de Cushing hipofisária-dependente, é uma doença endócrina rara e grave causada por um tumor hipofisário que produz ACTH excessivamente pelas células corticotróficas e está associada a aumento da morbidade e mortalidade. Ocorre mais frequentemente em mulheres (90\%), 3,5:1 e é de difícil diagnóstico. ${ }^{6}$

Os testes confirmatórios são: cortisol livre em urina de 24 horas; cortisol salivar no período noturno; teste de supressão com dexametasona; teste da dexametasona com hormônio liberador de corticotropina; e dosagem de ACTH. ${ }^{1,7}$ Exames de imagem como a ressonância nuclear magnética (RNM) da hipófise evidenciou adenoma em $35 \%$ a $60 \%$ dos pacientes. $^{7}$

No tratamento medicamentoso da HA na SC os inibidores da enzima conversora da angiotensina (IECA) ou bloqueadores dos receptores da angiotensina (BRA) devem ser os preferidos. ${ }^{1,8}$ assim como os diuréticos tiazídicos e a furosemida devem ser evitados, pois podem piorar uma eventual hipocalemia. ${ }^{1,8}$

Pode haver cura da HA com a remoção cirúrgica do tumor, porém 30\% dos pacientes mantêm HA sistólica e 25\% HA diastólica. ${ }^{8}$ Foi encontrada uma correlação entre a duração da HA antes da cirurgia e a persistência no pós-operatório. ${ }^{8}$

Nosso objetivo foi relatar um caso raro de HA secundária à SC, discutir a importância do diagnóstico etiológico precoce e o tratamento do hipercortisolismo que por sua vez podem melhorar o prognóstico com a reversão das comorbidades e fatores de risco cardiovascular.

\section{RELATO DE CASO}

Identificação: VNS, 23 anos, sexo feminino, branca, solteira, estudante, natural e procedente de São Paulo

Queixa principal

"Pressão alta" há 4 anos

\section{História}

Paciente encaminhada para consulta ambulatorial após ter sido atendida no Pronto-Socorro (PS) do Hospital São Paulo com quadro lá caracterizado como urgência hipertensiva. Na ocasião, apresentava cefaleia, tontura e níveis pressóricos elevados (210x110 mmHg). Relatava episódios de cefaleia holocraniana latejante de início gradual há 4 anos com piora há 2 anos, de forte intensidade com idas frequentes ao PS. Referia acompanhamento e uso regular de medicações anti-hipertensivas prescritas em Unidade Básica de Saúde (UBS). Referiu ainda aumento de peso, astenia e fraqueza muscular.

\section{Interrogatório sobre os diversos aparelhos}

Informou aumento de peso (mais de $25 \mathrm{~kg}$ em 2 anos), astenia, episódios frequentes de cefaleia, alterações visuais e insônia. Além disso, queixava-se de palpitações esporádicas e irregularidades menstruais há dois anos com períodos de amenorreia. Negava precordialgia, edema de membro inferior e dispneia paroxística noturna.

Medicações em uso regular

Hidroclorotiazida 25 mg/dia e losartana 50 mg de 12/12h.

\section{Antecedentes pessoais}

Menarca aos 9 anos. Hipertensão há 4 anos ( aos 18 anos de idade). Enxaqueca, colelitíase. Negava tabagismo, etilismo, uso de substâncias psicoativas, estimulantes, anti-inflamatórios não esteroidais ou anticoncepcionais orais. Referia sedentarismo e dieta sem restrições.

\section{Antecedentes familiares}

Pai hipertenso. Não possuía antecedentes de neoplasias na família.

\section{Exame fisico}

Bom estado geral, consciente, orientada, eupnéica, corada, hidratada, anictérica, fácies em lua cheia. Pele: estrias violáceas em abdome; acantose nigricans em região cervical.

Peso 105 kg, altura 1,60m, IMC $40 \mathrm{Kg} / \mathrm{m}^{2}$, circunferência abdominal $110 \mathrm{~cm}$.

PA em MSE (média de 2 aferições) 164/102 mm Hg, FC 95 bpm, FR 18 irpm.

Tórax: RCR2T, sem sopros audíveis. MV presente bilateral, sem ruídos adventícios. Abdome flácido e indolor, sem visceromegalias ou massas palpáveis, sem sopros e RHA presentes normais. Extremidades sem edemas e pulsos simétricos.

\section{Hipóteses diagnósticas}

Hipertensão arterial não controlada, obesidade grau III, síndrome de Cushing

\section{Conduta}

Realizado ECG no setor que mostrou ritmo sinusal sem 
alterações ou critérios para hipertrofia ventricular esquerda. Foi associado anlodipino $5 \mathrm{mg}$ a cada 12 horas ao esquema terapêutico inicial. Foram solicitados exames laboratoriais bioquímicos, monitorização ambulatorial da pressão arterial (MAPA) de 24 horas e exames para investigação de HA secundária. Foram orientadas medidas não farmacológicas (avaliação e acompanhamento nutricional reforçando orientações para perda de peso).

\section{Resultados de exames laboratoriais}

Hemograma normal; glicemia 92; HbA1c 6,4; ácido úrico 4,5; Na 142; K 5,0; uréia 20; creatinina 0,72; ClCr 106 $\mathrm{ml} / \mathrm{min} / 1,73^{3}$; CaT 9,7; TGO 21; TGP 40; CT 100; Tg 139; HDL 27; LDL 45; T4L 1,1; TSH 2,16; $\beta$-hcg negativo; Urina I sem alterações; aldosterona: $11,8 \mathrm{mg} / \mathrm{dl}(2,5-31)$ prolactina 56,3 ng/ml (3,8 - 28); GH 0,2; FSH 1,1; TT 0,33 ng/ml; LH 1,9; SDHEA 98,8. Cortisol urinário 24h 477 mg/24h (28,5$213,7)$; cortisol 8 h 25,9 (6,6 - 19,4); cortisol pós supressão $2 \mathrm{ug} / \mathrm{dl}$; ACTH (amostra 1) 23 pg/ml (até 46); ACTH (amostra 2) $90,9 \mathrm{pg} / \mathrm{ml}(7,2-63,3)$. (Tabela 1)

Tabela 1. Resultados da dosagem de cortisol urinário de 24h, dosagens plasmáticas de cortisol $8 \mathrm{~h}$ e cortisol após supressão e de ACTH plasmático.

\begin{tabular}{|c|c|c|}
\hline & Resultado & Referência \\
\hline Cortisol urinário (24h) & $477 \mathrm{mg} / 24 \mathrm{~h}$ & $28,5-213,7$ \\
\hline Cortisol 8h & $25,9 \mathrm{ug} / \mathrm{dl}$ & $6,6-19,4$ \\
\hline Cortisol após supressão & $2 \mathrm{ug} / \mathrm{dl}$ & - \\
\hline ACTH & $\begin{array}{c}23 \mathrm{pg} / \mathrm{ml} \text { (amostra 1) } \\
90,9 \mathrm{pg} / \mathrm{ml} \text { (amostra 2) }\end{array}$ & Até 46 \\
\hline
\end{tabular}

\section{ECOCARDIOGRAMA}

AO 28mm, AE 36mm, VD 18mm, SIV= PPVE 10mm, DDVE 45mm, DSVE 26 mm, Fração de Ejeção 73\%, Volume VE 92,45ml, IMVE 94,49 g/m², Espessura relativa da parede do VE 0,44\%. Cavidades Cardíacas com dimensões normais. Espessura e desempenho contrátil do VE globalmente conservados, valvas atrioventriculares com morfologia normal, refluxo valvar mitral de grau mínimo e tricúspide de grau leve, PSAP $31 \mathrm{mmHg}$, arco aórtico sem particularidades, pericárdio sem alterações, veia cava inferior com diâmetro normal e variação respiratória preservada; velocidade máxima do refluxo tricúspide: $2,5 \mathrm{~m} / \mathrm{s}$.

USG rins e vias urinárias e doppler de artérias renais

Dentro dos padrões da normalidade.

\section{TC abdome}

Dentro dos padrões da normalidade. RNM

Lesão expansiva adeno-hipofisária compatível com macroadenoma. Nodulação com hipersinal em T1 no extremo lateral direito do interior selar, inespecífica, mas que pode corresponder a neurohipófise deslocada. (Figura 1)

\section{EVOLUÇÃO}

A MAPA 24 horas realizada em uso de HCT $25 \mathrm{mg}$ ao dia, losartana $50 \mathrm{mg}$ a cada 12 horas e anlodipino $5 \mathrm{mg}$ a cada
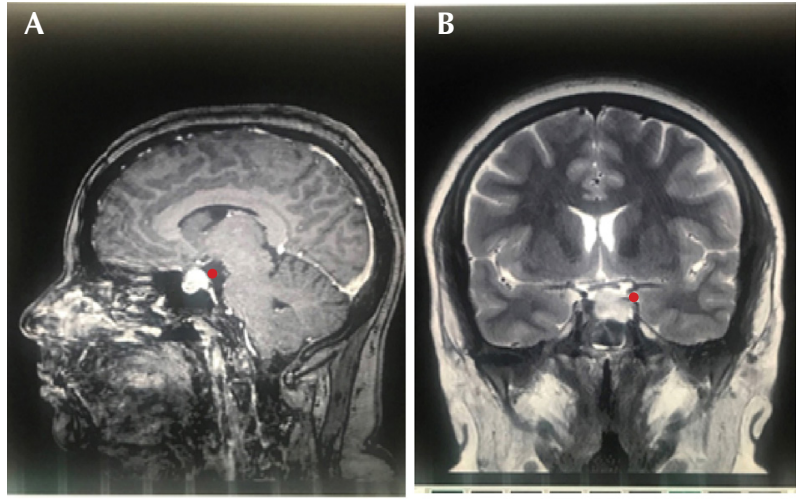

Figura 1. Lesão expansiva adeno-hipofisária compatível com macroadenoma, como indicado pela seta. Nodulação com hipersinal em T1 no extremo lateral direito do interior selar, inespecífica, mas que pode corresponder a neurohipófise deslocada. A - RNM de sela túrcica na projeção sagital. B - RNM de sela túrcica em projeção coronal.

12 horas confirmou HAR (Figura 2A) secundária a doença de Cushing (Figura 1) foi introduzida a espironolactona $25 \mathrm{mg}$ ao dia no esquema terapêutico.

Após adoção de medidas não farmacológicas e acompanhamento nutricional houve perda de $12 \mathrm{Kg}$ do peso inicial.

Optou-se inicialmente pela introdução de cetoconazol (200 mg 2x/dia) e posteriormente 200 mg 3x/dia sem melhora clínica e/ou imagem após 6 meses. Foi indicada e submetida a cirurgia por abordagem transesfenoidal cujo resultado cirúrgico foi considerado satisfatório.

Realizada avaliação clínica no setor de Cardiopatia Hipertensiva depois de 3 meses do procedimento, a paciente encontrava-se assintomática e ao exame físico estava em bom estado geral, hidratada, normocorada, anictética, afebril, ausência de estrias violáceas ou fascies cushingoide. Pesava $93 \mathrm{~kg}$, circunferência abdominal 94cm, IMC 36,3 kg/m2. A PA (mm Hg) média de 2 medidas em MSE 129/78, FC 82 bpm, FR 19 irpm. Ausculta cardíaca e pulmonar normais, abdome flácido, indolor à palpação e sem visceromegalias. As extremidades não evidenciaram edemas e os pulsos eram rítmicos e simétricos.

Os exames laboratoriais realizados posteriormente estavam dentro dos limites normais. A MAPA 24 horas realizada após procedimento evidenciou medidas pressóricos normais (Figura 2B).

\section{DISCUSSÃO}

A SC é uma afecção causada por exposição prolongada a níveis excessivos de cortisol ou outros glicocorticoides de fontes endógenas ou exógenas. É caracterizada clinicamente por obesidade na parte superior do corpo, hipertensão arterial, diabetes, osteoporose, hirsutismo, amenorreia e excesso de líquido corporal. A SC endógena ou hipercortisolismo espontâneo é dividido em dois grupos, aqueles causados por excesso de hormônio ACTH e os que são independentes do ACTH. A DC é uma doença da hipófise caracterizada pelo aumento da secreção do ACTH o que leva à hipersecreção de cortisol pelas glândulas suprarrenais resultando na SC. A DC endógena é rara e de difícil diagnóstico.

A HA é uma manifestação frequente da SC e está presente 


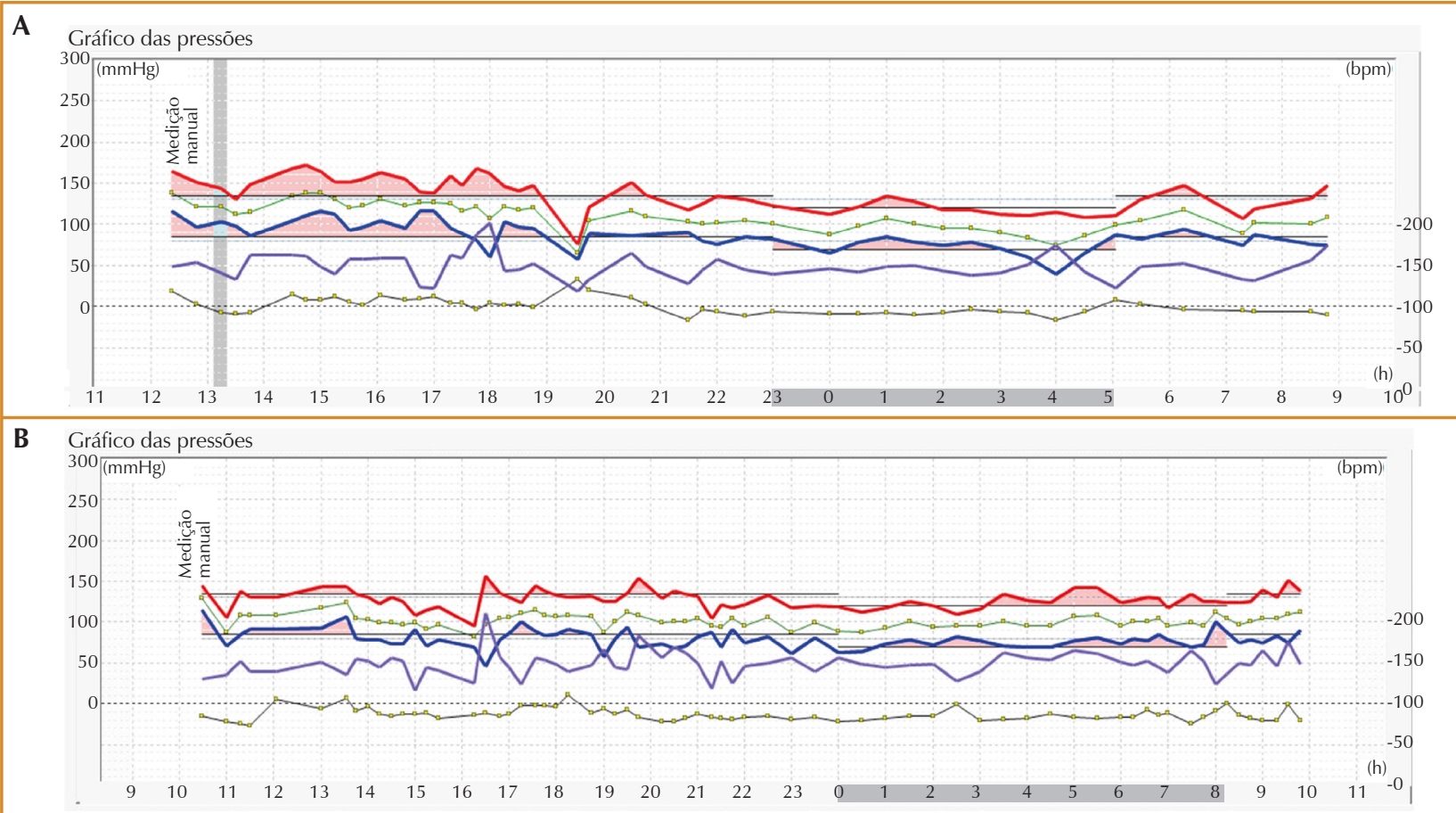

Figura 2. A - Gráfico dos valores pressóricos sistólicos/diastólicos $(\mathrm{mm} \mathrm{Hg})$ pela MAPA realizada antes do procedimento cirúrgico: período total:137/87, vigília 142/92, sono 118/71, ascensão matinal $18 \mathrm{~mm} \mathrm{Hg}$, descenso do sono (\%) 17\%/23\%, carga pressórica (\%): vigília 68\%/73\%, sono $36 \% / 64 \%$, total $60 \% / 71 \%$. B - Gráfico dos valores pressóricos sistólicos/diastólicos ( $\mathrm{mm} \mathrm{Hg}$ ) pela MAPA após procedimento cirúrgico: período total 128/79, vigília 129/81, sono 125/76, ascensão matinal $18 \mathrm{~mm} \mathrm{Hg}$, descenso do sono (\%) 3\%/6\%, cargas pressóricas (\%): vigília 30\%/32\%, sono $65 \% / 75 \%$, total $40 \% / 45 \%$.

em $80 \%$ dos casos. $^{2}$ Ela surge precocemente e é um fator de risco para mortalidade, ${ }^{3}$ daí a importância de se fazer este diagnóstico. Da mesma forma deve-se pensar em SC principalmente no aparecimento de hipertensão em jovens e em casos de HAR.

A idade da paciente e os níveis pressóricos apresentados chamaram a atenção para uma investigação minuciosa do caso e foi realizada a monitorização ambulatorial da pressão arterial (MAPA) de 24 h que confirmou a presença de HAR. ${ }^{9}$ Somando-se a isto, os dados clínicos da anamnese (cefaleia, alterações visuais, insônia), alterações de exame físico (facie Cushingoide, obesidade central, giba, estrias violáceas abdominais) e laboratoriais sugeriram fortemente a possibilidade da síndrome de Cushing e foi iniciada a investigação desta condição.

Estabelecer a causa da síndrome de Cushing é um dos processos mais desafiadores da clínica. Testes bioquímicos, incluindo dosagem do ACTH plasmático, supressão de dexametasona em altas doses e teste de estímulo do hormônio liberador de corticotropina, são essenciais para o diagnóstico diferencial. No entanto, nenhum teste existente tem precisão diagnóstica suficiente quando usado sozinho. $\mathrm{O}$ uso adjuvante de imagens focadas, incluindo tomografia computadorizada, RNM e modalidades de imagens nucleares, geralmente pode fornecer um diagnóstico. Em pacientes com SC dependente de $\mathrm{ACTH}$, a amostragem bilateral do seio petroso inferior pode facilitar o diagnóstico em pessoas com características clínicas, bioquímicas ou de imagens discrepantes. ${ }^{10}$

No nosso caso, o quadro de hipercortisolismo foi estabelecido e feita a diferenciação entre SC dependente de ACTH e não dependente de ACTH. O hipercortisolismo foi confirmado por meio de dosagem do ACTH plasmático em duas amostras que estavam aumentadas $(>15 \mathrm{pg} / \mathrm{mL})$ e configurou-se a SC dependente de ACTH, responsável pela maioria dos casos (80\%). ${ }^{11}$ Diante desta confirmação laboratorial seguiu-se a investigação quanto a sua possível etiologia. Como a maioria destes (90\%) tem como causa a doença de Cushing por adenoma hipofisário foi realizada a investigação por método de imagem. ${ }^{11}$

Apesar da investigação com RNM fazer-se necessária, em mais de $40 \%$ dos casos o exame pode não ser capaz de evidenciar o adenoma. ${ }^{12} \mathrm{O}$ diagnóstico neste caso foi realizado com base nos exames laboratoriais e pela RNM que foi conclusiva quanto à presença de macroadenoma hipofisário e afastando a possibilidade de produção ectópica de ACTH na SC.

O tratamento da SC é essencial para reduzir a mortalidade e as comorbidades associadas. O tratamento eficaz inclui a normalização dos níveis de cortisol ou sua ação. Também inclui a normalização de comorbidades por meio do tratamento direto da causa da SC e de tratamentos adjuvantes (por exemplo, anti-hipertensivos). A ressecção cirúrgica da (s) lesão (ões) causal (s) geralmente é a abordagem de primeira linha. A escolha dos tratamentos de segunda linha, incluindo medicamentos, adrenalectomia bilateral e radioterapia (para tumores com corticotrópicos), deve ser individualizada para cada paciente.

A ressecção cirúrgica da(s) lesão (ões) pode ser seguida por 
remissão da doença em cerca de $78 \%$ e recidiva em cerca de $13 \%$ dos pacientes durante o período de 10 anos após a cirurgia, de modo que quase um terço dos pacientes experimenta a longo prazo uma falha da cirurgia e requer um tratamento de segunda linha adicional. Pacientes com DC persistente ou recorrente requerem tratamentos adicionais, incluindo radioterapia hipofisária, cirurgia adrenal e/ou terapia clínica. A radioterapia hipofisária é eficaz no controle do excesso de cortisol em uma grande porcentagem de pacientes, mas está associada a um risco considerável de hipopituitarismo. A adrenalectomia bilateral é seguida de um controle rápido e definitivo do excesso de cortisol em quase todos os pacientes, mas induz a insuficiência adrenal.

A terapia clínica adquiriu recentemente um papel mais importante em relação ao passado, devido ao uso recente de novos compostos capazes de controlar a secreção ou ação do cortisol. Atualmente, a terapia clínica é usada como tratamento pré-cirúrgico, principalmente para doenças graves; ou como tratamento pós-cirúrgico, nos casos de falha ou ressecção cirúrgica incompleta do tumor; ou como terapia de transição antes, durante e após a radioterapia enquanto espera o controle da doença; ou, em casos selecionados, como terapia primária, principalmente quando a cirurgia não é uma opção. O cetoconazol, dirigido para a adrenal, é o fármaco mais utilizado, principalmente por sua rápida ação, enquanto o antagonista do receptor de glicocorticoide, o mifepristone, é altamente eficaz no controle de comorbidades clínicas, principalmente a intolerância à glicose, sendo, portanto, um tratamento útil para DC quando é associada a diabetes. Os medicamentos direcionados à hipófise têm a vantagem de atuar no local responsável pela DC, o tumor hipofisário. Entre esse grupo de drogas, o agonista da dopamina cabergolina e o análogo da somatostatina pasireotida resultam na remissão da doença em um subgrupo consistente de pacientes com DC. O pasireotido pode ser usado para o tratamento da DC quando a cirurgia falhou ou quando a cirurgia não é uma opção, e o mifepristone para o tratamento da SC quando associado ao comprometimento do metabolismo da glicose em caso de falta de indicação cirúrgica. A experiência recente sugere que a combinação de diferentes drogas pode ser capaz de controlar o excesso de cortisol na grande maioria dos pacientes com DC. ${ }^{13}$

Neste caso, a paciente foi tratada com cetoconazol por seis meses. O cetoconazol é um dos medicamentos mais prescritos para o tratamento da DC, apesar de ser usado off label. Este composto é um fármaco antifúngico imidazol que inibe reversivelmente a esteroidogênese adrenal por meio da ação de várias enzimas (isto é, colesterol desmolase, $17 \mathrm{aOH}$ e 11b-hidroxilase). O cetoconazol também inibe a produção de andrógenos, resultando em hipogonadismo em homens (ginecomastia, diminuição da libido e disfunção erétil); em mulheres, ao contrário, pode melhorar o hiperandrogenismo. ${ }^{14}$ Os efeitos colaterais leves são relativamente comuns e incluem dor de cabeça, náuseas e erupção na pele. Outro efeito colateral importante é o aumento dos níveis de transaminases hepáticas em até três vezes o limite superior. Esse aumento geralmente é assintomático e reversível com a interrupção do medicamento ou redução da dose. Portanto, é importante monitorar as transaminases hepáticas durante o primeiro mês de tratamento e depois disso. Insuficiência hepática idiossincrática grave foi descrita em raras ocasiões. ${ }^{14}$
É um medicamento que pode oferecer uma taxa de controle que varia entre 30 e $80 \% .{ }^{15}$

A ressecção cirúrgica é a melhor e única forma de tratamento definitivo da doença de Cushing ${ }^{15}$ e possui altas taxas de remissão inicial relatadas após a intervenção que variaram de $69 \%$ a $98 \%$. Porém Shepard et al. encontraram taxas de recorrência que variaram de $7 \%$ e $66 \%{ }^{16}$

A hipertensão é uma das características relevantes da DC endógena pois está presente em cerca de $80 \%$ dos pacientes adultos e em cerca de $47 \%$ de crianças. A hipertensão na DC está significativamente correlacionada com a duração do hipercortisolismo e resulta da interação entre vários mecanismos fisiopatológicos que regulam o volume plasmático, a resistência vascular periférica e o débito cardíaco, todos aumentados nesse estado. Os glicocorticoides causam hipertensão por vários mecanismos: sua atividade mineralocorticoide intrínseca; pela ativação do sistema renina-angiotensina; pelo aumento de substâncias vasoativas e pela supressão dos sistemas vasodilatadores. Além disso, os glicocorticoides podem exercer alguns efeitos hipertensivos na regulação cardiovascular pelo sistema nervoso central (SNC) por meio dos receptores de glicocorticóides e mineralocorticoides.

A hipertensão na DC geralmente se resolve com a remoção cirúrgica do tumor, mas os pacientes requerem tratamento anti-hipertensivo farmacológico no pré e algumas vezes no pós-operatório. As tiazidas e a furosemida devem ser evitadas, enquanto o bloqueio adrenérgico e os antagonistas dos canais de cálcio são geralmente ineficazes. Os IECA, BRA e antagonistas dos receptores mineralocorticoides, são boas opções anti-hipertensivas; os agonistas do PPAR- $\gamma$ podem ajudar em muitos aspectos da síndrome de resistência à insulina e o antagonista do receptor de glicocorticoide, relativamente seletivo, mifepristone (RU 486) pode reduzir a pressão arterial em pacientes com DC. Agentes neuromoduladores como os inibidores da serotonina ciproheptadina e ritanserina, ácido valproide, agonistas da dopamina, análogos da somatostatina podem ocasionalmente ser eficazes, assim como drogas que atuam diretamente nos níveis adrenais, como o cetoconazol. ${ }^{8}$

A paciente foi abordada cirurgicamente e após 38 meses do início da investigação evoluiu com normalização dos níveis pressóricos no consultório e à MAPA (vigília) sem uso de medicações antihipertensivas, assim como melhora do perfil metabólico laboratorial. (Figura 2B)

A adoção de medidas comportamentais saudáveis e atividade física orientadas e acompanhadas durante o seguimento ambulatorial da paciente levaram a mudança de hábitos de vida e contribuiram para a perda de peso, o que certamente teve impacto no controle pressórico, mas a retirada da medicação anti-hipertensiva só se deu após a retirada cirúrgica do adenoma.

Com a remoção cirúrgica do tumor pode haver cura da HA, porém 30\% dos pacientes mantêm HA sistólica e 25\% HA diastólica. ${ }^{8}$ Foi encontrada uma correlação entre a duração da HA antes da cirurgia e a persistência no pós-operatório. ${ }^{8}$

O acompanhamento dos níveis pressóricos após ressecção cirúrgica devem portanto ser monitorados e caso necessário a reintrodução dos agentes anti-hipertensivos.

O tratamento da hipertensão na DC continua sendo uma tarefa difícil e um grande desafio, a fim de diminuir a morbimortalidade associada à doença. 


\section{REFERÊNCIAS}

1. Malachias MVB, Souza WKSB, Plavnik FL, et al. 7a Diretriz Brasileira de Hipertensão Arterial. Arq Bras Cardiol. 2016; 107(3Supl.3):1-83.

2. Newell-Price J. Diagnosis/differential diagnosis of Cushing's syndrome: a review of best practice. Best Pract Res Clin Endocrinol Metab. 2009;23 Suppl 1:S5-14. doi: 10.1016/S1521-690X(09)70003-X

3. Isidori AM, Graziadio Ch, Paragliola RM, et al., ABC Study Group. The hypertension of Cushing's syndrome: controversies in the pathophysiology and focus on cardiovascular complications. J Hypertens. 2015;33(1):44-60. doi: 10.1097/HJH.0000000000000415.

4. Arnaldi G, Angeli A, Atkinson AB, et al. Diagnosis and complications of Cushing's syndrome: a consensus statement. J Clin Endocrinol Metab. 2003;88(12):5593-602. doi: 10.1210/jc.2003-030871.

5. Lindsay JR, Nieman LK. The hypothalamic-pituitary-adrenal axis in pregnancy: challenges in disease detection and treatment. Endocr Rev. 2005;26(6):775-99. doi: 10.1210/er.2004-0025.

6. Cuevas-Ramos D, Ting Lim DS, Fleseriu M. Update on medical treatment for Cushing's disease - Clin Diabetes Endocrinol. 2016;2:16. doi: 10.1186/ s40842-016-0033-9.

7. Prevedello DM, Challinor SM, Tomycz ND, Kassam A. Diagnosing, managing Cushing's disease: a multidisciplinary overview. Review of Endocrinology. 2009;1:19-24.

8. Cicala MV, Mantero F. Hypertension in Cushing's syndrome: from pathogenesis to treatment. Neuroendocrinology. 2010;92 Suppl 1:44-9. doi: $10.1159 / 000314315$
9. de La Sierra A, Segura J, Banegas JR, et al. Clinical features of 8295 patients with resistant hypertension classified on the basis of ambulatory blood pressure monitoring. Hypertension. 2011;57(5):898-902.

10. Lindsay JR, Nieman LK. Differential diagnosis and imaging in Cushing's syndrome. Endocrinol Metab Clin North Am. 2005;34(2):403-21, x. doi: 10.1016/j.ecl.2005.01.009.

11. Tritos NA, Biller BMK. Current management of Cushing's disease. J Intern Med. 2019 ; 286 (5): 526-541. doi: 10.1111/joim.12975.

12. Bertagna X, Guignat L, Groussin L, Bertherat J. Cushing's disease. Best Pract Res Clin Endocrinol Metab. 2009;23(5):607-23. doi:10.1016/j. beem.2009.06.001.

13. Pivonello R, De Leo M, Cozzolino A, Colao A. The Treatment of Cushing's Disease. Endocr Rev. 2015;36(4):385-486. doi: 10.1210/er.2013-1048.

14. Machado MC, Fragoso MCBV, Moreira AC, et al. A review of Cushing's disease treatment by the Department of Neuroendocrinology of the Brazilian Society of Endocrinology and Metabolism. Arch Endocrinol Metab. 2018;62/1. Doi: 10.20945/2359-3997000000014

15. Nieman LK, Biller BM, Findling JW, et al. Treatment of Cushing's Syndrome: An Endocrine Society Clinical Practice Guideline. J Clin Endocrinol Metab. $2015 ; 100(8): 2807-31$. doi: 10.1210/jc.2015-1818.

16. Shepard MJ, Mehta GU, Xu Z, et al. Technique of Whole-Sellar Stereotactic Radiosurgery for Cushing Disease: Results from a Multicenter, International Cohort Study. World Neurosurg. 2018 Aug;116:e670-e679. doi: 10.1016/j. wneu.2018.05.067. 